\title{
Neutrosophic Set - A Generalization of the Intuitionistic Fuzzy Set
}

\author{
Florentin SMARANDACHE \\ University of New Mexico \\ 705 Gurley Ave. \\ Gallup, NM 87301, USA \\ E-mail:smarand@unm.edu
}

\begin{abstract}
In this paper one generalizes the intuitionistic fuzzy set (IFS), paraconsistent set, and intuitionistic set to the neutrosophic set (NS). Many examples are presented. Distinctions between NS and IFS are underlined.

Keywords and Phrases: Intuitionistic Fuzzy Set, Paraconsistent Set, Intuitionistic Set, Neutrosophic Set, Non-standard Analysis, Philosophy.

MSC 2000: 03B99, 03E99.

\section{Introduction.}

One first presents the evolution of sets from fuzzy set to neutrosophic set. Then one introduces the neutrosophic components T, I, F which represent the membership, indeterminacy, and non-membership values respectively, where $]^{-} 0,1^{+}[$is the non-standard unit interval, and thus one defines the neutrosophic set. One gives examples from mathematics, physics, philosophy, and applications of the neutrosophic set. Afterwards, one introduces the neutrosophic set operations (complement, intersection, union, difference, Cartesian product, inclusion, and n-ary relationship), some generalizations and comments on them, and finally the distinctions between the neutrosophic set and the intuitionistic fuzzy set.
\end{abstract}

\section{Short History.}


The fuzzy set (FS) was introduced by L. Zadeh [1] in 1965, where each element had a degree of membership (t).

The intuitionistic fuzzy set (IFS) on a universe $\mathrm{X}$ was introduced by K. Atanassov [2] in 1986 as a generalization of FS, where besides the degree of membership $\mu_{A}(x) \in[0,1]$ of each element $x \in X$ to a set A there was considered a degree of non-membership $v_{A}(x) \in[0,1]$, but such that

$$
\forall x \in X \mu_{A}(x)+v_{A}(x) \leq 1
$$

The neutrosophic set (NS) was introduced by F. Smarandache [3] who introduced the degree of indeterminacy (i) as indepedent component in his 1995 manuscris that was published in 1998.

According to Deschrijver \& Kerre (2003) the vague set defined by Gau and Buehrer (1993) was proven by Bustine \& Burillo (1996) to be the same as IFS.

Goguen (1967) defined the $L$-fuzzy Set in $X$ as a mapping $X \rightarrow L$ such that $\left(L^{*}, \leq_{L^{*}}\right)$ is a complete lattice, where $L^{*}=\left\{\left(x_{1}, x_{2}\right) \in[0,1]^{2}, x_{1}+x_{2} \leq 1\right\}$ and $\left(x_{1}, x_{2}\right) \leq_{L^{*}}\left(y_{1}, y_{2}\right) \Leftrightarrow$ $x_{1} \leq y_{1}$ and $x_{2} \geq y_{2}$. The interval-valued fuzzy sets (IVFS) apparently first studied by Sambuc (1975), which were called by Deng (1989) grey sets, and IFS are specific kinds of L-fuzzy sets.

According to Cornelis et al. (2003), Gehrke et al. (1996) stated that "Many people believe that assigning an exact number to an expert's opinion is too restrictive, and the assignment of an interval of values is more realistic", which is somehow similar with the imprecise probability theory where instead of a crisp probability one has an interval (upper and lower) probabilities as in Walley (1991).

Atanassov (1999) defined the interval-valued intuitionistic fuzzy set (IVIFS) on a universe $\mathrm{X}$ as an object $\mathrm{A}$ such that:

$$
\begin{aligned}
& A=\left\{\left(x, M_{A}(X), N_{A}(x)\right), x \in X\right\}, \\
& \text { with } M_{A}: X \rightarrow \operatorname{Int}([0,1]) \text { and } N_{A}: X \rightarrow \operatorname{Int}([0,1]), \\
& \text { and } \forall x \in X \sup M_{A}(x)+\sup N_{A}(x) \leq 1 .
\end{aligned}
$$

Belnap (1977) defined a four-valued logic, with truth (T), false (F), unknown (U), and contradiction $(\mathrm{C})$. He used a billatice where the four components were inter-related. 
In 1995, starting from philosophy (when I fretted to distinguish between absolute truth and relative truth or between absolute falsehood and relative falsehood in logics, and respectively between absolute membership and relative membership or absolute nonmembership and relative non-membership in set theory) I began to use the non-standard analysis. Also, inspired from the sport games (winning, defeating, or tie scores), from votes (pro, contra, null/black votes), from positive/negative/zero numbers, from yes/no/NA, from decision making and control theory (making a decision, not making, or hesitating), from accepted/rejected/pending, etc. and guided by the fact that the law of excluded middle did not work any longer in the modern logics, I combined the non-standard analysis with a tricomponent logic/set/probability theory and with philosophy (I was excited by paradoxism in science and arts and letters, as well as by paraconsistency and incompleteness in knowledge). How to deal with all of them at once, is it possible to unity them?

I proposed the term "neutrosophic" because "neutrosophic" etymologically comes from "neutrosophy" [French neutre < Latin neuter, neutral, and Greek sophia, skill/wisdom] which means knowledge of neutral thought, and this third/neutral represents the main distinction between "fuzzy"/ "intuitionistic fuzzy" logic/set and „,neutrosophic” logic/set, i.e. the included middle component (Lupasco-Nicolescu's logic in philosophy), i.e. the neutral/indeterminate/unknown part (besides the "truth"/"membership" and "falsehood"/"nonmembership" components that both appear in fuzzy logic/set).

See the Proceedings of the First International Conference on Neutrosophic Logic, The University of New Mexico, Gallup Campus, 1-3 December 2001, at:

http://fs.gallup.unm.edu/FirstNeutConf.htm .

\section{Definition of Neutrosophic Set.}

Let T, I, F be real standard or non-standard subsets of $]^{-} 0,1^{+}[$,

with $\sup T=t_{-} \sup , \inf T=t_{-} i n f$,

$$
\sup I=i_{-} \sup , \inf I=i_{-} i n f \text {, }
$$$$
\sup F=f_{-} \text {sup, } \inf F=f_{-} \text {inf },
$$

and $n_{-}$sup $=t \_s u p+i \_s u p+f \_s u p$, 


$$
n_{-} i n f=t_{-} i n f+i_{-} i n f+f \_i n f \text {. }
$$

T, I, F are called neutrosophic components.

Let $\mathrm{U}$ be a universe of discourse, and $\mathrm{M}$ a set included in $\mathrm{U}$. An element $x$ from $\mathrm{U}$ is noted with respect to the set $\mathrm{M}$ as $x(T, I, F)$ and belongs to $\mathrm{M}$ in the following way: it is $t \%$ true in the set, $i \%$ indeterminate (unknown if it is) in the set, and $f \%$ false, where $t$ varies in $\mathrm{T}$, $i$ varies in $\mathrm{I}, f$ varies in $\mathrm{F}$.

For software engineering proposals the classical unit interval $[0,1]$ is used.

For single valued neutrosophic logic $(\mathrm{t}, \mathrm{i}, \mathrm{f})$, the sum of the components is:

$0 \leq \mathrm{t}+\mathrm{i}+\mathrm{f} \leq 3$ when all three components are independent;

$0 \leq \mathrm{t}+\mathrm{i}+\mathrm{f} \leq 2$ when two components are dependent, while the third one is independent from them;

$0 \leq \mathrm{t}+\mathrm{i}+\mathrm{f} \leq 1$ when all three components are dependent.

When three or two of the components $t, i, f$ are independent, one leaves room for incomplete information (sum < 1), paraconsistent and contradictory information ( information $(\mathrm{sum}=1)$.

In general [3], the sum of two components $\mathrm{x}$ and $\mathrm{y}$ that vary in the unitary interval $[0,1]$ is:

$0 \leq x+y \leq 2-d^{\circ}(x, y)$, where $d^{\circ}(x, y)$ is the degree of dependence between $x$ and $y$.

Therefore $2-d^{\circ}(x, y)$ is the degree of independence between $x$ and $y$.

\section{General Examples.}

Let $\mathrm{A}, \mathrm{B}$, and $\mathrm{C}$ be three neutrosophic sets.

One can say, by language abuse, that any element neutrosophically belongs to any set, due to the percentages of truth/indeterminacy/falsity involved, which varies between 0 and 1 , or even less than 0 or greater than 1 .

Thus: $x(0.5,0.2,0.3)$ belongs to $\mathrm{A}$ (which means, with a probability of $50 \% x$ is in $\mathrm{A}$, with a probability of $30 \% x$ is not in $\mathrm{A}$, and the rest is undecidable); or $y(0,0,1)$ belongs to $\mathrm{A}$ (which normally means $y$ is not for sure in A); or $z(0,1,0)$ belongs to A (which means one does know absolutely nothing about $z$ 's affiliation with $\mathrm{A}$ ); herein $0.5+0.2+0.3=1$; thus $\mathrm{A}$ is a 
$\mathrm{NS}$ and an IFS too. More general, $y((0.20-0.30),(0.40-0.45) \cup[0.50-$ $0.51],\{0.20,0.24,0.28\})$ belongs to the set $\mathrm{B}$, which means:

- with a probability in between $20-30 \% y$ is in B (one cannot find an exact approximation because of various sources used);

- with a probability of $20 \%$ or $24 \%$ or $28 \% y$ is not in B;

- the indeterminacy related to the appurtenance of $y$ to B is in between $40-45 \%$ or between $50-51 \%$ (limits included).

The subsets representing the appurtenance, indeterminacy, and falsity may overlap, and $n_{-}$sup $=0.30+0.51+0.28>1$ in this case; then B is a NS but is not an IFS; we can call it paraconsistent set (from paraconsistent logic, which deals with paraconsistent information).

Or, another example, say the element $z(0.1,0.3,0.4)$ belongs to the set $\mathrm{C}$, and here $0.1+0.3+0.4<1 ;$ then B is a NS but is not an IFS; we can call it intuitionistic set (from intuitionistic logic, which deals with incomplete information).

Remarkably, in the same NS one can have elements which have paraconsistent information (sum of components $>1$ ), others incomplete information (sum of components $<$ 1 ), others consistent information (in the case when the sum of components $=1$ ), and others interval-valued components (with no restriction on their supremum or infimum sums).

\section{Physics Examples.}

a) For example the Schrödinger's Cat Theory says that the quantum state of a photon can basically be in more than one place in the same time, which translated to the neutrosophic set means that an element (quantum state) belongs and does not belong to a set (one place) in the same time; or an element (quantum state) belongs to two different sets (two different places) in the same time. It is a question of "alternative worlds" theory, very well represented by the neutrosophic set theory.

In Schrödinger's Equation on the behavior of electromagnetic waves and "matter waves" in quantum theory, the wave function $\psi$ which describes the superposition of possible states may be simulated by a neutrosophic function, i.e. a function whose values are not unique for each argument from the domain of definition (the vertical line test fails, intersecting the graph in more points). 
Don't we better describe, using the attribute "neutrosophic" than "fuzzy" or "intuitionistic fuzzy" or any others, a quantum particle that neither exists nor non-exists?

b) How to describe a particle $\zeta$ in the infinite micro-universe that belongs to two distinct places $P_{1}$ and $P_{2}$ in the same time? $\zeta \in P_{1}$ and $\zeta \notin P_{1}$ as a true contradiction, or $\zeta \in P_{1}$ and $\zeta \in$ $\neg P_{1}$.

\section{Philosophical Examples.}

Or, how to calculate the truth-value of Zen (in Japanese) / Chan (in Chinese) doctrine philosophical proposition: the present is eternal and comprises in itself the past and the future?

In Eastern Philosophy the contradictory utterances form the core of the Taoism and Zen/Chan (which emerged from Buddhism and Taoism) doctrines.

How to judge the truth-value of a metaphor, or of an ambiguous statement, or of a social phenomenon which is positive from a standpoint and negative from another standpoint?

There are many ways to construct them, in terms of the practical problem we need to simulate or approach. Below there are mentioned the easiest ones.

\section{Application.}

A cloud is a neutrosophic set, because its borders are ambiguous, and each element (water drop) belongs with a neutrosophic probability to the set (e.g. there are a kind of separated water drops, around a compact mass of water drops, that we don't know how to consider them: in or out of the cloud).

Also, we are not sure where the cloud ends nor where it begins, neither if some elements are or are not in the set. That's why the percent of indeterminacy is required and the neutrosophic probability (using subsets - not numbers - as components) should be used for better modelling: it is a more organic, smooth, and especially accurate estimation. Indeterminacy is the zone of ignorance of a proposition's value, between truth and falsehood.

\section{Operations with classical Sets.}


We need to present these set operations in order to be able to introduce the neutrosophic connectors. Let $S_{1}$ and $S_{2}$ be two (unidimensional) real standard or non-standard subsets included in the non-standard interval $\left.]^{-} 0, \infty\right)$, and $a \in R$ a real number, then one defines:

\subsection{Addition of classical Sets:}

$S_{1} \oplus S_{2}=\left\{x \mid x=s_{1}+s_{2}\right.$, where $s_{1} \in S_{1}$ and $\left.s_{2} \in S_{2}\right\}$,

with $\inf S_{1} \oplus S_{2}=\inf S_{1}+\inf S_{2}, \sup S_{1} \oplus S_{2}=\sup S_{1}+\sup S_{2}$;

and, as some particular cases, we have

$$
\{a\} \oplus S_{2}=\left\{x \mid x=a+s_{2}, \text { where } s_{2} \in S_{2}\right\}
$$

with $\inf \{a\} \oplus S_{2}=a+\inf S_{2}, \sup \{a\} \oplus S_{2}=a+\sup S_{2}$.

\subsection{Subtraction of classical Sets:}

$S_{1} \ominus S_{2}=\left\{x \mid x=s_{1}-s_{2}\right.$, where $s_{1} \in S_{1}$ and $\left.s_{2} \in S_{2}\right\}$,

with $\inf S_{1} \ominus S_{2}=\inf S_{1}-\inf S_{2}, \sup S_{1} \ominus S_{2}=\sup S_{1}-\sup S_{2}$;

and, as some particular cases, we have

$\{a\} \ominus S_{2}=\left\{x \mid x=a-s_{2}\right.$, where $\left.s_{2} \in S_{2}\right\}$,

with $\inf \{a\} \ominus S_{2}=a-\inf S_{2}, \sup \{a\} \ominus S_{2}=a-\sup S_{2}$;

also $\left\{1^{+}\right\} \ominus S_{2}=\left\{x \mid x=1^{+}-s_{2}\right.$, where $\left.s_{2} \in S_{2}\right\}$,

with $\inf \left\{1^{+}\right\} \ominus S_{2}=1^{+}-\sup S_{2}, \sup \left\{1^{+}\right\} \ominus S_{2}=100-\inf S_{2}$.

\subsection{Multiplication of classical Sets:}

$$
S_{1} \odot S_{2}=\left\{x \mid x=s_{1} \cdot s_{2} \text {, where } s_{1} \in S_{1} \text { and } s_{2} \in S_{2}\right\} \text {. }
$$

with inf $S_{1} \odot S_{2}=\inf S_{1} \cdot \inf S_{2}, \sup S_{1} \odot S_{2}=\sup S_{1} \cdot \sup S_{2}$;

and, as some particular cases, we have

$\{a\} \odot S_{2}=\left\{x \mid x=a \cdot s_{2}\right.$, where $\left.s_{2} \in S_{2}\right\}$, 
with $\inf \{a\} \odot S_{2}=a * \inf S_{2}, \sup \{a\} \odot S_{2}=a \cdot \sup S_{2}$;

also $\left\{1^{+}\right\} \odot S_{2}=\left\{x \mid x=1 \cdot s_{2}\right.$, where $\left.s_{2} \in S_{2}\right\}$,

with $\inf \left\{1^{+}\right\} \odot S_{2}=1^{+} \cdot \inf S_{2}, \sup \left\{1^{+}\right\} \odot S_{2}=1^{+} \cdot \sup S_{2}$.

\subsection{Division of a Classical Set by a Number:}

Let $k \in \mathbb{R}^{*}$, then $S_{1} \oslash k=\left\{x \mid x=s_{1} / k\right.$, where $\left.s_{1} \in S_{1}\right\}$.

\section{Classical Logic and Set, Fuzzy Logic and Set, Intuitionistic Fuzzy Logic and Set, and Neutrosophic Logic and Set.}

In order to make distinction between classical (Boolean) logic/set, fuzzy logic/set, intuitionistic fuzzy logic/set, and neutrosophic logic/set, we denote their corresponding operators (negation/complement, conjunction/intersection, disjunction/union, implication, and equivalence), as it follows:

a. For classical (Boolean) logic and set:

$$
\neg \wedge \vee \rightarrow \leftrightarrow
$$

b. For fuzzy logic and set:

$$
\begin{array}{lllll}
\neg & \wedge & \vee & \rightarrow & \leftrightarrow \\
F & F & F & F & F
\end{array}
$$

c. For intuitionistic fuzzy logic and set:

$$
\begin{array}{ccccc}
\neg & \wedge & \vee & \rightarrow & \leftrightarrow \\
I F & I F & I F & I F & I F
\end{array}
$$

d. For neutrosophic logic and set:

$$
\begin{array}{ccccc}
\neg & \wedge & \vee & \rightarrow & \leftrightarrow \\
N & N & N & N & N
\end{array}
$$

10. Neutrosophic Set Operations. 
One notes, with respect to the sets $\mathrm{A}$ and $\mathrm{B}$ over the universe $\mathrm{U}, x=x\left(T_{1}, I_{1}, F_{1}\right) \in A$ and $x=x\left(T_{2}, I_{2}, F_{2}\right) \in B$, by mentioning $x$ 's neutrosophic membership, indeterminacy, and non-membership respectively appurtenance.

And, similarly, $y=y\left(T^{\prime}, I^{\prime}, F^{\prime}\right) \in B$.

If, after calculations, in the below operations one obtains values $<0$ or $>1$, then one replaces them with ${ }^{-} 0$ or $1^{+}$respectively.

\subsection{Complement of $A$ :}

There are defined in neutrosophic literature classes of neutrosophic negation operators as follows: if $A\left(t_{A}, i_{A}, f_{A}\right)$, then its negation is:

$$
\begin{aligned}
& \text { or }{ }_{N} A\left(f_{A}, i_{A}, t_{A}\right) \text {, } \\
& \text { or }{ }_{N} A\left(f_{A}, 1-i_{A}, t_{A}\right) \text {, } \\
& \text { or }{ }_{N}^{A}\left(1-t_{A}, 1-i_{A}, 1-f_{A}\right) \text {, } \\
& \text { or } ᄀ_{N} A\left(1-t_{A}, i_{A}, 1-f_{A}\right) \text {, }
\end{aligned}
$$

etc.

\subsection{Intersection:}

Similarly, classes of neutrosophic conjunctive operators:

if $A\left(t_{A}, i_{A}, f_{A}\right)$ and $B\left(t_{B}, i_{B}, f_{B}\right)$, then

$$
\begin{aligned}
& A \stackrel{\wedge}{N} B=\left\langle t_{A} \stackrel{\wedge}{F} t_{B}, i_{A} \stackrel{\vee}{F} i_{B}, f_{A} \stackrel{\vee}{F} f_{B}\right\rangle, \\
& \text { or } A_{N}^{\wedge} B=\left\langle t_{A} \stackrel{\wedge}{F} t_{B}, i_{A} \stackrel{\wedge}{F} i_{B}, f_{A} \stackrel{\vee}{F} f_{B}\right\rangle, \\
& \text { or } A{ }_{N}^{\wedge} B=\left\langle t_{A} \stackrel{\wedge}{F} t_{B}, i_{A} \stackrel{\wedge}{F} i_{B}, f_{A} \stackrel{\wedge}{F} f_{B}\right\rangle, \\
& \text { or } A{ }_{N}^{\wedge} B=\left\langle t_{A} \stackrel{\wedge}{F} t_{B}, \frac{i_{A}+i_{B}}{2}, f_{A} \underset{F}{\vee} f_{B}\right\rangle,
\end{aligned}
$$


or $A \stackrel{\wedge}{\wedge} B=\left\langle t_{A} \stackrel{\wedge}{F} t_{B}, 1-\frac{i_{A}+i_{B}}{2}, f_{A} \stackrel{\vee}{\vee} f_{B}\right\rangle$,

or $A_{N}^{\wedge} B=\left\langle t_{A} \hat{F} t_{B},\left|i_{A}-i_{B}\right|, f_{A} \stackrel{\vee}{F} f_{B}\right\rangle$, etc.

\subsection{Union:}

And, analogously, classes of neutrosophic disjunctive operators were defined:

$$
\begin{aligned}
& A_{N}^{\vee} B=\left\langle t_{A} \stackrel{\vee}{\vee} t_{B}, i_{A} \stackrel{\wedge}{i_{B}}, f_{A} \hat{F} f_{B}\right\rangle, \\
& \text { or } A_{N}^{\vee} B=\left\langle t_{A} \stackrel{\vee}{\vee} t_{B}, i_{A} \vee \underset{F}{\vee} i_{B}, f_{A} \hat{F} f_{B}\right\rangle \text {, } \\
& \text { or } A_{N}^{\vee} B=\left\langle t_{A} \underset{F}{\vee} t_{B}, i_{A} \underset{F}{\vee} i_{B}, f_{A}^{\vee v} f_{B}\right\rangle \text {, } \\
& \text { or } A_{N}^{\vee} B=\left\langle t_{A} \stackrel{\vee}{F} t_{B}, \frac{i_{A}+i_{B}}{2}, f_{A} \hat{F} f_{B}\right\rangle \text {, } \\
& \text { or } A \stackrel{\vee}{\vee} B=\left\langle t_{A} \stackrel{\vee}{\vee} t_{B}, 1-\frac{i_{A}+i_{B}}{2}, f_{A} \hat{F} f_{B}\right\rangle \text {, } \\
& \text { or } A_{N}^{\vee} B=\left\langle t_{A} \stackrel{\vee}{\vee} t_{B},\left|i_{A}-i_{B}\right|, f_{A} \stackrel{\vee}{\vee} f_{B}\right\rangle \text {, etc. }
\end{aligned}
$$

\subsection{Difference:}

Because $A \backslash B=A \cap C(B)$, the difference can be defined using any of the above neutrosophic intersection and neutrosophic negation operator.

\subsection{Cartesian Product:}

If $x\left(T_{1}, I_{1}, F_{1}\right) \in A, y\left(T^{\prime}, I^{\prime}, F^{\prime}\right) \in B$, then $\left(x\left(T_{1}, I_{1}, F_{1}\right), y\left(T^{\prime}, I^{\prime}, F^{\prime}\right)\right) \in A \times B$.

\subsection{A is a subset of $B$ :}

If $x\left(T_{1}, I_{1}, F_{1}\right) \in A \Rightarrow x\left(T_{2}, I_{2}, F_{2}\right) \in B$, where $\inf T_{1} \leq \inf T_{2}, \sup T_{1} \leq \sup T_{2}$, and $\inf F_{1} \geq \inf F_{2}, \sup F_{1} \geq \sup F_{2}$. 


\subsection{Neutrosophic n-ary Relation.}

Let $A_{1}, A_{2}, \ldots, A_{n}$ be arbitrary non-empty sets.

A Neutrosophic n-ary Relation $R$ on $A_{1} \times A_{2} \times \ldots \times A_{n}$ is defined as a subset of the Cartesian product $A_{1} \times A_{2} \times \ldots \times A_{n}$, such that for each ordered n-tuple $\left(x_{1}, x_{2}, \ldots, x_{n}\right)(T, I, F)$, $T$ represents the degree of validity, $I$ the degree of indeterminacy, and $F$ the degree of nonvalidity respectively of the relation $R$.

It is related to the definitions for the Intuitionistic Fuzzy Relation independently given by Atanassov (1984, 1989), Toader Buhaescu (1989), Darinka Stoyanova (1993), Humberto Bustince Sola and P. Burillo Lopez (1992-1995).

\subsection{The Neutrosophic Quantifiers.}

The Neutrosophic Existential Quantifier is in the following way:

There exist $x\left(t_{x}, i_{x}, f_{x}\right)$ in A such that $P(x)\left(t_{P}, i_{P}, f_{P}\right)$

or

$$
\exists x\left(t_{x}, i_{x}, f_{x}\right) \in A, P(x)\left(t_{P}, i_{P}, f_{P}\right) ;
$$

which means that: there exists an element $\mathrm{x}$ which belongs to A in a neutrosophic degree $\left(t_{\mathrm{x}}\right.$, $\left.i_{x}, f_{x}\right)$, such that the proposition $P$ has the neutrosophic degree of truth $\left(t_{P}, i_{P}, f_{P}\right)$.

The Neutrosophic Universal Quantifier is the following way:

For any $\mathrm{x}\left(\mathrm{t}_{\mathrm{x}}, \mathrm{i}_{\mathrm{x}}, \mathrm{f}_{\mathrm{x}}\right)$ in $\mathrm{A}$ one has $\mathrm{P}(\mathrm{x})\left(\mathrm{t}_{\mathrm{P}}, \mathrm{i}_{\mathrm{P}}, \mathrm{f}_{\mathrm{P}}\right)$

or

$$
\forall x\left(t_{x}, i_{x}, f_{x}\right) \in A, P(x)\left(t_{P}, i_{P}, f_{P}\right) ;
$$

which means that: for any element $x$ that belongs to $A$ in a neutrosophic degree $\left(t_{x}, i_{x}, f_{x}\right)$, one has the proposition $\mathrm{P}$ with the neutrosophic degree of truth $\left(t_{\mathrm{P}}, \mathrm{i}_{\mathrm{P}}, \mathrm{f}_{\mathrm{P}}\right)$.

\subsection{Classes of Neutrosophic Implications.}

Whence, since $(A \underset{N}{\rightarrow} B) \underset{N}{\leftrightarrow}\left(\neg_{N} A \underset{N}{\vee} B\right)$, one also has Classes of Neutrosophic Implication Operators as $\left({ }_{N} A \stackrel{\vee}{\vee} B\right)$, where the neutrosophic negation can be any of the above definitions, and the neutrosophic disjunction, similarly any of the above definitions.

\subsubsection{Fuzzy Operators.}


Let $\alpha, \beta \in[0,1]$.

The fuzzy negation has been defined as ${ }_{F} \alpha=1-\alpha$, while the class of fuzzy conjunctions (or t-norm) may be:

$$
\alpha_{F}^{\wedge} \beta=\min \{\alpha, \beta\}
$$

or $\alpha_{F}^{\wedge} \beta=\alpha \cdot \beta$,

or $\alpha_{F}^{\wedge} \beta=\max \{0, \alpha+\beta-1\}$, etc.

And the class of fuzzy disjunctions (or t-conorm) may be:

$$
\alpha_{F}^{\vee} \beta=\max \{\alpha, \beta\}
$$

or $\alpha_{F}^{\vee} \beta=\alpha+\beta-\alpha \beta$,

or $\alpha_{F}^{\vee} \beta=\min \{1, \alpha+\beta\}$, etc.

Examples of fuzzy implications $x \underset{F}{\rightarrow} y$, for $x, y \in[0,1]$, defined below:

- Fodor (1993): $I_{F D}(x, y)=\left\{\begin{array}{c}1, \text { if } x \leq y \\ \max (1-x, y), \text { if } x>y\end{array}\right.$

- Weber (1983): $I_{W B}(x, y)=\left\{\begin{array}{l}1, \text { if } x<y \\ y, \text { if } x=1\end{array}\right.$

- Yager (1980): $I_{Y G}(x, y)=\left\{\begin{array}{l}1, \text { if } x=0 \text { and } y=0 \\ y^{x}, \text { if } x>0 \text { or } y>0\end{array}\right.$

- Goguen (1969): $I_{G G}(x, y)=\left\{\begin{array}{l}1, \text { if } x \leq y \\ \frac{y}{x}, \text { if } x>y\end{array}\right.$

- Rescher (1969): $I_{R S}(x, y)=\left\{\begin{array}{l}1, \text { if } x \leq y \\ 0, \text { if } x>y\end{array}\right.$

- Kleene-Dienes (1938): $I_{K D}(x, y)=\max (1-x, y)$

- Reichenbach (1935): $I_{R C}(x, y)=1-x+x y$

- Gödel (1932): $I_{G D}(x, y)=\left\{\begin{array}{l}1, \text { if } x \leq y \\ y, \text { if } x>y\end{array}\right.$

- Lukasiewicz (1923): $I_{L K}(x, y)=\min (1,1-x+y)$,

according to Michal Baczyński and Balasubramaniam Jayaram (2008).

10.9.2. Example of intuitionistic fuzzy implications $A\left(t_{A}, f_{A}\right) \underset{I F}{\rightarrow} B\left(t_{B}, f_{B}\right)$ is: 


$$
I_{I F}=\left(\left[\left(1-t_{A}\right)_{F}^{\vee} t_{B}\right]_{F}^{\wedge}\left[\left(1-f_{B}\right)_{F}^{\vee} f_{A}\right], f_{B}^{\wedge}\left(1-t_{A}\right)\right),
$$

according to Yunhua Xiao, Tianyu Xue, Zhan'ao Xue, and Huiru Cheng (2011).

10.9.3. We now propose eight classes of neutrosophic implications:

$$
A\left(t_{A}, i_{A}, f_{A}\right) \underset{N}{\rightarrow} B\left(t_{B}, i_{B}, f_{B}\right),
$$

in the following ways:

1-2. $I_{N 1}\left(t_{A} \underset{F / I F}{\longrightarrow} t_{B}, i_{A F} \hat{F} i_{B}, f_{A F} \hat{F} f_{B}\right)$, where $t_{A} \underset{F / I F}{\longrightarrow} t_{B}$ is any of the above fuzzy implications of intuitionistic fuzzy implications, while ${ }_{F}^{\wedge}$ is any fuzzy conjunction;

3-4. $I_{N 2}\left(t_{A} \underset{F / I F}{\longrightarrow} t_{B}, i_{A F}^{\vee} i_{B}, f_{A F} \wedge f_{B}\right)$, where $\underset{F}{\vee}$ is any fuzzy disjunction;

5-6. $I_{N 3}\left(t_{A} \underset{F / I F}{\longrightarrow} t_{B}, \frac{i_{A}+i_{B}}{2}, f_{A F} \wedge f_{B}\right)$;

7-8. $I_{N 4}\left(t_{A} \underset{F / I F}{\longrightarrow} t_{B}, \frac{i_{A}+i_{B}}{2}, \frac{f_{A}+f_{B}}{2}\right)$

Let's see an example of neutrosophic implication.

Let's have two neutrosophic propositions $A\langle 0.3,0.4,0.2\rangle$ and $B\langle 0.7,0.1,0.4\rangle$. Then $A$ $\underset{N}{\rightarrow} B$ has the neutrosophic truth value of ${ }_{N} A_{N}^{\vee} B$, i.e.:

$\langle 0.2,0.4,0.3\rangle_{N}^{\vee}\langle 0.7,0.1,0.4\rangle$

or $\langle\max \{0.2,0.7\}, \min \{0.4,0.1\}, \min \{0.3,0.4\}\rangle$,

Or $\langle 0.7,0.1,0.3\rangle$,

where we used the neutrosophic operators defined above:

${ }_{N}\langle t, i, f\rangle=\langle f, i, t\rangle$ for neutrosophic negation

and $\left\langle t_{1}, i_{1}, f_{1}\right\rangle_{N}^{\vee}\left\langle t_{2}, i_{2}, f_{2}\right\rangle=\left\langle\max \left\{t_{1}, t_{2}\right\}, \min \left\{i_{1}, i_{2}\right\}, \min \left\{f_{1}, f_{2}\right\}\right\rangle$ for the neutrosophic disjunction.

Using different versions of the neutrosophic negation operators and/or different versions of the neutrosophic disjunction operators, one obtains, in general, different results. Similarly as in fuzzy logic. 
Another example for neutrosophic implication.

Let $A$ have the neutrosophic truth value $\left(t_{A}, i_{A}, f_{A}\right)$, and $B$ have the neutrosophic truth value $\left(t_{B}, i_{B}, f_{B}\right)$, then:

$$
[A \underset{N}{\rightarrow} B] \stackrel{\leftrightarrow}{\leftrightarrow}\left[\left({ }_{N} A\right)_{N}^{\vee} B\right]
$$

where $\vec{N}$ is any of the above neutrosophic negations, while $\underset{N}{v}$ is any of the above neutrosophic disjunctions.

We consider that the most general definition of neutrosophic operators shall be the followings:

$$
\begin{gathered}
A\left(t_{A}, i_{A}, f_{A}\right) \oplus_{N}^{\oplus} B\left(t_{B}, i_{B}, f_{B}\right)=A_{N}^{\oplus} B\left\langle u\left(t_{A}, i_{A}, f_{A}, t_{B}, i_{B}, f_{B}\right), \quad v\left(t_{A}, i_{A}, f_{A}, t_{B}, i_{B}, f_{B}\right),\right. \\
\left.w\left(t_{A}, i_{A}, f_{A}, t_{B}, i_{B}, f_{B}\right)\right\rangle
\end{gathered}
$$

where $\underset{N}{\oplus}$ is any binary neutrosophic operator, and $u\left(x_{1}, x_{2}, x_{3}, x_{4}, x_{5}, x_{6}\right), v\left(x_{1}, x_{2}, x_{3}, x_{4}, x_{5}, x_{6}\right), w\left(x_{1}, x_{2}, x_{3}, x_{4}, x_{5}, x_{6}\right):[0,1]^{6} \rightarrow[0,1]$

Even more, the neutrosophic component functions $u, v, w$ may depend, on the top of these six variables, on hidden parameters as well, such as: $h_{1}, h_{2}, \ldots, h_{n}$.

For a unary neutrosophic operator (for example, the neutrosophic negation), similarly:

$$
{ }_{N}^{\urcorner} A\left(t_{A}, i_{A}, f_{A}\right)=\left\langle u^{\prime}\left(t_{A}, i_{A}, f_{A}\right), v^{\prime}\left(t_{A}, i_{A}, f_{A}\right), w^{\prime}\left(t_{A}, i_{A}, f_{A}\right)\right\rangle
$$

where $u^{\prime}\left(t_{A}, i_{A}, f_{A}\right), v^{\prime}\left(t_{A}, i_{A}, f_{A}\right), w^{\prime}\left(t_{A}, i_{A}, f_{A}\right):[0,1]^{3} \rightarrow[0,1]$,

and even more $u^{\prime}, v^{\prime}, w^{\prime}$ may depend, on the top of these three variables, of hidden parameters as well, such as: $h_{1}, h_{2}, \ldots, h_{n}$.

As an example, we have defined in F. Smarandache, V. Christianto, n-ary Fuzzy Logic and Neutrosophic Logic Operators, published in Studies in Logic Grammar and Rhetoric, Belarus, 17(30), pp. 1-16, 2009:

$$
A\left(t_{A}, i_{A}, f_{A}\right){ }_{N}^{\wedge} B\left(t_{B}, i_{B}, f_{B}\right)=\left\langle t_{A} t_{B}, i_{A} i_{B}+t_{A} i_{B}+t_{B} i_{A}, t_{A} f_{B}+t_{B} f_{A}+i_{A} f_{B}+i_{B} f_{A}\right\rangle
$$

these result from multiplying

$$
\left(t_{A}+i_{A}+f_{A}\right) \cdot\left(t_{B}+i_{B}+f_{B}\right)
$$


and ordering upon the below pesimistic order:

$$
\text { truth } \prec \text { indeterminacy } \prec \text { falsity, }
$$

meaning that to the truth only the terms of $t$ 's goes, i.e. $t_{A} t_{B}$,

to indeterminacy only the terms of t's and i's go, i.e. $i_{A} i_{B}+t_{A} i_{B}+t_{B} i_{A}$,

and to falsity the other terms left, i.e. $t_{A} f_{B}+t_{B} f_{A}+i_{A} f_{B}+i_{B} f_{A}+f_{A} f_{B}$.

\section{Generalizations and Comments.}

From the intuitionistic logic, paraconsistent logic, dialetheism, faillibilism, paradoxes, pseudoparadoxes, and tautologies we transfer the "adjectives" to the sets, i.e. to intuitionistic set (set incompletely known), paraconsistent set, dialetheist set, faillibilist set (each element has a percentage of indeterminacy), paradoxist set (an element may belong and may not belong in the same time to the set), pseudoparadoxist set, and tautologic set respectively.

Hence, the neutrosophic set generalizes:

- the intuitionistic set, which supports incomplete set theories (for $0<n<1$ and $i=0,0 \leq t, i, f \leq 1)$ and incomplete known elements belonging to a set;

- the fuzzy set (for $\mathrm{n}=1$ and $\mathrm{i}=0$, and $0 \leq t, i, f \leq 1$ );

- the intuitionistic fuzzy set (for $t+i+f=1$ and $0 \leq i<1$ );

- the classical set (for $n=1$ and $i=0$, with $t, f$ either 0 or 1 );

- the paraconsistent set (for $n>1$ and $i=0$, with both $t, f<1$ ); there is at least one element $x(T, I, F)$ of a paraconsistent set $\mathrm{M}$ which belongs at the same time to $\mathrm{M}$ and to its complement set $\mathrm{C}(\mathrm{M})$;

- the faillibilist set $(i>0)$;

- the dialethist set, which says that the intersection of some disjoint sets is not empty (for $t=f=1$ and $i=0$; some paradoxist sets can be denoted this way too); every element $x(T, I, F)$ of a dialethist set $\mathrm{M}$ belongs at the same time to $\mathrm{M}$ and to its complement set $\mathrm{C}(\mathrm{M})$;

- the paradoxist set, each element has a part of indeterminacy if it is or not in the set $(i>1)$;

- the pseudoparadoxist set $(0<i<1, t+f>1)$;

- the tautological set $(i<0)$. 
Compared with all other types of sets, in the neutrosophic set each element has three components which are subsets (not numbers as in fuzzy set) and considers a subset, similarly to intuitionistic fuzzy set, of "indeterminacy" - due to unexpected parameters hidden in some sets, and let the superior limits of the components to even boil over 1 (overflooded) and the inferior limits of the components to even freeze under $O$ (underdried).

For example: an element in some tautological sets may have $t>1$, called "overincluded". Similarly, an element in a set may be "overindeterminate" (for $i>1$, in some paradoxist sets), "overexcluded" (for $f>1$, in some unconditionally false appurtenances); or "undertrue" (for $t<0$, in some unconditionally false appurtenances), "underindeterminate" (for $i<0$, in some unconditionally true or false appurtenances), "underfalse" (for $f<0$, in some unconditionally true appurtenances).

This is because we should make a distinction between unconditionally true $(t>1$, and $f<0$ or $i<0)$ and conditionally true appurtenances $(t \leq 1$, and $f \leq 1$ or $i \leq 1)$.

In a rough set $\mathrm{RS}$, an element on its boundary-line cannot be classified neither as a member of RS nor of its complement with certainty. In the neutrosophic set a such element may be characterized by $x(T, I, F)$, with corresponding set-values for $T, I, F \subseteq]^{-} 0,1^{+}[$.

Compared to Belnap's quadruplet logic, NS and NL do not use restrictions among the components - and that's why the NS/NL have a more general form, while the middle component in NS and NL (the indeterminacy) can be split into more subcomponents if necessarily in various applications.

\section{Distinctions between Neutrosophic Set (NS) and Intuitionistic Fuzzy Set (IFS).}

1) Neutrosophic Set can distinguish between

- absolute membership (i.e. membership in all possible worlds; we have extended Leibniz's absolute truth to absolute membership), and

- relative membership (membership in at least one world, but not in all), because NS (absolute membership element) $=1^{+}$

while

- $\quad$ NS (relative membership element) $=1$. 
This has application in philosophy (see the neutrosophy). That's why the unitary standard interval $[0,1]$ used in IFS has been extended to the unitary non-standard interval ]$^{-} 0,1^{+}[$in NS.

Similar distinctions for absolute or relative non-membership, and absolute or relative indeterminant appurtenance are allowed in NS.

2) In NS, there is no restriction on T, I, F other than they are subsets of $]^{-} 0,1^{+}[$, thus: ${ }^{-} 0 \leq \inf \mathrm{T}+\inf \mathrm{I}+\inf \mathrm{F}\left[\sup \mathrm{T}+\sup \mathrm{I}+\sup \mathrm{F} \leq 3^{+}\right.$.

The inequalities (2.1) and (2.4) of IFS are relaxed in NS. 
This non-restriction allows paraconsistent, dialetheist, and incomplete information to be characterized in NS \{i.e. the sum of all three components if they are defined as points, or sum of superior limits of all three components if they are defined as subsets can be $>1$ (for paraconsistent information coming from different sources), or $<1$ for incomplete information \}, while that information cannot be described in IFS because in IFS the components $\mathrm{T}$ (membership), I (indeterminacy), $\mathrm{F}$ (non-membership) are restricted either to $\mathrm{t}+\mathrm{i}+\mathrm{f}=1$ or to $\mathrm{t}^{2}+\mathrm{f}^{2} \leq 1$, if $\mathrm{T}, \mathrm{I}, \mathrm{F}$ are all reduced to the points $\mathrm{t}, \mathrm{i}, \mathrm{f}$ respectively, or to sup $\mathrm{T}+$ $\sup \mathrm{I}+\sup \mathrm{F}=1$ if $\mathrm{T}, \mathrm{I}, \mathrm{F}$ are subsets of $[0,1]$. Of course, there are cases when paraconsistent and incomplete informations can be normalized to 1 , but this procedure is not always suitable.

This most important distinction between IFS and NS is showed in the below Neutrosophic Cube A'B'C'D'E'F'G'H' introduced by J. Dezert in 2002.

Because in technical applications only the classical interval $[0,1]$ is used as range for the neutrosophic parameters $t, i, f$, we call the cube $A B C D E D G H$ the technical neutrosophic cube and its extension $A^{\prime} B^{\prime} C^{\prime} D^{\prime} E^{\prime} D^{\prime} G^{\prime} H^{\prime}$ the neutrosophic cube (or absolute neutrosophic cube), used in the fields where we need to differentiate between absolute and relative (as in philosophy) notions.

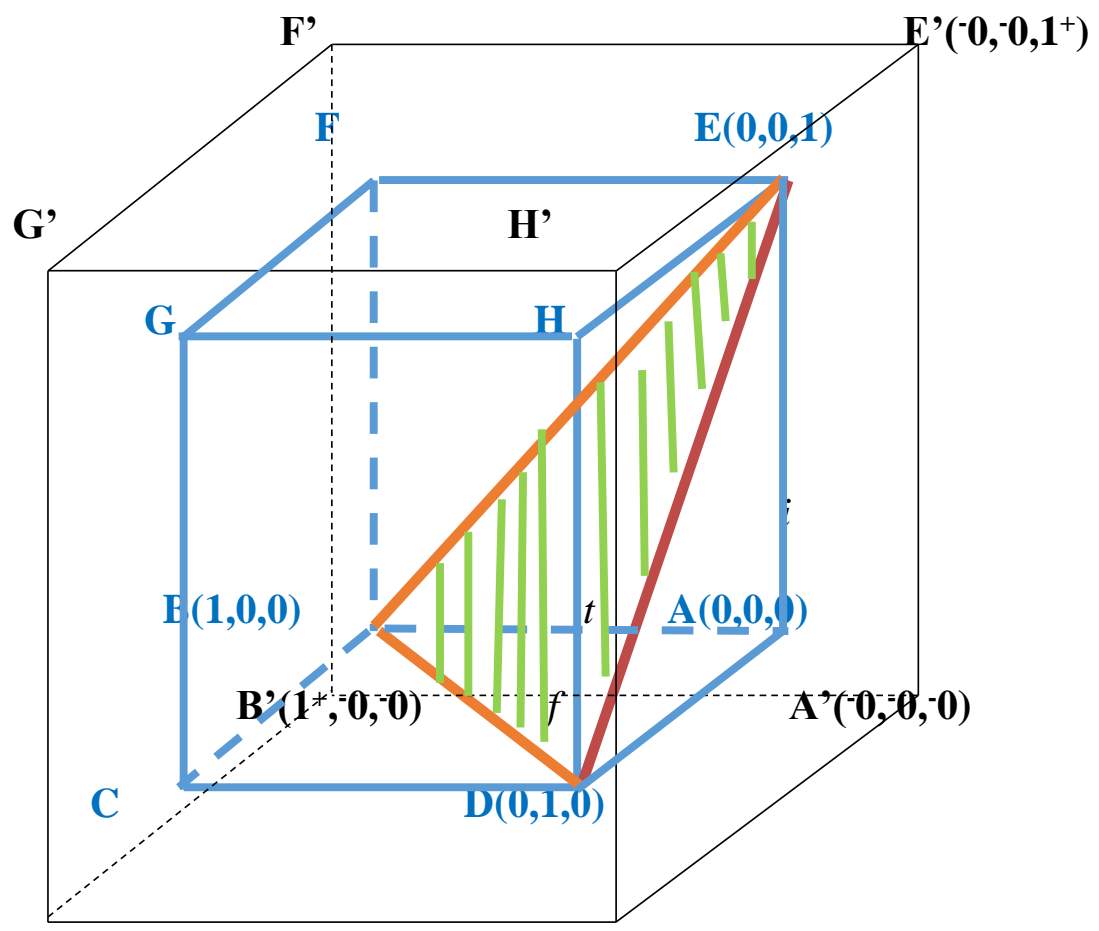

$C^{\prime}$

$\mathbf{D}^{\prime}\left(\mathbf{- 0 , \mathbf { 1 } ^ { + } , \mathbf { - 0 } )}\right.$ 
Let's consider a 3D Cartesian system of coordinates, where $t$ is the truth axis with value range in $]^{-} 0,1^{+}[, f \text { is the false axis with value range in }]^{-} 0,1^{+}[$, and similarly $i$ is the indeterminate axis with value range in $]^{-} 0,1^{+}[$. regions:

We now divide the technical neutrosophic cube $A B C D E D G H$ into three disjoint

a) The equilateral triangle $B D E$, whose sides are equal to $\sqrt{2}$, which represents the geometrical locus of the points whose sum of the coordinates is 1 .

If a point $Q$ is situated on the sides of the triangle $B D E$ or inside of it, then $t_{Q}+i_{Q}+f_{Q}=1$ as in Atanassov-intuitionistic fuzzy set $(A-I F S)$.

b) The pyramid $E A B D$ \{ situated in the right side of the $\triangle E B D$, including its faces $\triangle A B D$ (base), $\triangle E B A$, and $\triangle E D A$ (lateral faces), but excluding its face $\triangle B D E\}$ is the locus of the points whose sum of coordinates is less than 1 .

If $P \in E A B D$ then $t_{P}+i_{P}+f_{P}<1$ as in intuitionistic set (with incomplete information).

c) In the left side of $\triangle B D E$ in the cube there is the solid EFGCDEBD ( excluding $\triangle B D E$ ) which is the locus of points whose sum of their coordinates is greater than 1 as in the paraconsistent set.

If a point $R \in E F G C D E B D$, then $t_{R}+i_{R}+f_{R}>1$.

It is possible to get the sum of coordinates strictly less than 1 or strictly greater than 1 . For example having three independent sources of information:

- We have a source which is capable to find only the degree of membership of an element; but it is unable to find the degree of non-membership;

- Another source which is capable to find only the degree of non-membership of an element;

- Or a source which only computes the indeterminacy.

Thus, when we put the results together of these sources, it is possible that their sum is not 1 , but smaller or greater.

Also, in information fusion, when dealing with indeterminate models (i.e. elements of the fusion space which are indeterminate/unknown, such as intersections we don't know if they are empty or not since we don't have enough information, similarly for complements of indeterminate elements, etc.): if we compute the believe in that element (truth), the disbelieve in that element (falsehood), and the indeterminacy part of that element, then the sum of these three components is strictly less than 1 (the difference to 1 is the missing information).

3) Relation (2.3) from interval-valued intuitionistic fuzzy set is relaxed in NS, i.e. the intervals do not necessarily belong to $\operatorname{Int}[0,1]$ but to $[0,1]$, even more general to $]^{-} 0,1^{+}[$.

4) In NS the components T, I, F can also be non-standard subsets included in the unitary 
nonstandard interval $] 0,1^{+}[$, not only standard subsets included in the unitary standard interval $[0,1]$ as in IFS.

5) NS, like dialetheism, can describe paradoxist elements, $\mathrm{NS}$ (paradoxist element $)=(1, \mathrm{I}$, 1), while IFL cannot describe a paradox because the sum of components should be 1 in IFS.

6) The connectors in IFS are defined with respect to T and F only, i.e. membership and nonmembership only (hence the Indeterminacy is what's left from 1), while in NS they can be defined with respect to any of them (no restriction).

But, for interval-valued intuitionistic fuzzy set one cannot find any left indeterminacy.

7) Component " $I$ ", indeterminacy, can be split into more subcomponents in order to better catch the vague information we work with, and such, for example, one can get more accurate answers to the Question-Answering Systems initiated by Zadeh (2003).

\{In Belnap's four-valued logic (1977) indeterminacy is split into Uncertainty $(U)$ and Contradiction $(C)$, but they were interrelated.

Even more, one can split "I" into Contradiction, Uncertainty, and Unknown, and we get a five-valued logic.

In a general Refined Neutrosophic Logic, $T$ can be split into subcomponents $T_{1}, T_{2}, \ldots, T_{p}$, and $I$ into $I_{1}, I_{2}, \ldots, I_{r}$, and $F$ into $F_{1}, F_{2}, \ldots, F_{s}$, where $p, r, s \geq 1$ and $p+r+s=n \geq 3$. Even more: $T$, I, and/or $F$ (or any of their subcomponents $T_{j}, I_{k}$, and/or $F_{l}$ ) can be countable or uncountable infinite sets.

8) Indeterminacy is independent from membership/truth and non-membership/falsehood in $\mathrm{NS} / \mathrm{Nl}$, while in IFS/IFL it is not.

In neutrosophics there are two types of indeterminacies:

a) Numerical Indeterminacy (or Degree of Indeterminacy), which has the form $(t, i, f)$ $\neq(1,0,0)$, where $t, i, f$ are numbers, intervals, or subsets included in the unit interval $[0,1]$, and it is the base for the $(t, i, f)$-Neutrosophic Structures.

b) Non-numerical Indeterminacy (or Literal Indeterminacy), which is the letter " $P$ " standing for unknown (non-determinate), such that $I^{2}=I$, and used in the composition of the neutrosophic number $N=a+b I$, where $a$ and $b$ are real or complex numbers, and $a$ is the determinate part of number $N$, while $b I$ is the indeterminate part of $N$. The neutrosophic numbers are the base for the I-Neutrosophic Structures.

9) NS has a better and clear terminology (name) as "neutrosophic" (which means the neutral part: i.e. neither true/membership nor false/nonmembership), while IFS's name "intuitionistic" produces confusion with Intuitionistic Logic, which is something different (see the article by Didier Dubois et al., 2005).

10) The Neutrosophic Set was extended [Smarandache, 2007] to Neutrosophic Overset (when some neutrosophic component is >1), and to Neutrosophic Underset (when some neutrosophic component is < 0), and to and to Neutrosophic Offset (when some neutrosophic 
components are off the interval $[0,1]$, i.e. some neutrosophic component $>1$ and some neutrosophic component $<0$ ).

This is no surprise with respect to the classical fuzzy set/logic, intuitionistic fuzzy set/logic, or classical and imprecise probability where the values are not allowed outside the interval [0, 1], since our real-world has numerous examples and applications of over/under/off neutrosophic components.

Example:

In a given company a full-time employer works 40 hours per week. Let's consider the last week period.

Helen worked part-time, only 30 hours, and the other 10 hours she was absent without payment; hence, her membership degree was $30 / 40=0.75<1$.

John worked full-time, 40 hours, so he had the membership degree $40 / 40=1$, with respect to this company.

But George worked overtime 5 hours, so his membership degree was $(40+5) / 40=45 / 40=$ $1.125>1$. Thus, we need to make distinction between employees who work overtime, and those who work full-time or part-time. That's why we need to associate a degree of membership greater than 1 to the overtime workers.

Now, another employee, Jane, was absent without pay for the whole week, so her degree of membership was $0 / 40=0$.

Yet, Richard, who was also hired as a full-time, not only didn't come to work last week at all ( 0 worked hours), but he produced, by accidentally starting a devastating fire, much damage to the company, which was estimated at a value half of his salary (i.e. as he would have gotten for working 20 hours). Therefore, his membership degree has to be less that Jane's (since Jane produced no damage). Whence, Richard's degree of membership with respect to this company was $-20 / 40=-0.50<0$.

Therefore, the membership degrees $>1$ and $<0$ are real in our world, so we have to take them into consideration.

Then, similarly, the Neutrosophic Logic/Measure/Probability/Statistics etc. were extended to respectively Neutrosophic Over/Under/Off Logic, Measure, Probability, Statistics etc.

[Smarandache, 2007].

11) Neutrosophic Tripolar (and in general Multipolar) Set and Logic [Smarandache, 2007] of the form:

$\left(\left\langle\mathrm{T}^{+}{ }_{1}, \mathrm{~T}^{+}{ }_{2}, \ldots, \mathrm{T}^{+}{ }_{\mathrm{n}} ; \mathrm{T}^{0} ; \mathrm{T}_{-\mathrm{n}}^{-}, \ldots, \mathrm{T}^{-}{ }_{-2}, \mathrm{~T}_{-1}^{-}\right\rangle,\left\langle\mathrm{I}^{+}{ }_{1}, \mathrm{I}^{+}{ }_{2}, \ldots, \mathrm{I}^{+}{ }_{\mathrm{n}} ; \mathrm{I}^{0} ; \mathrm{I}_{-\mathrm{n}}^{-}, \ldots, \mathrm{I}_{-2}^{-}, \mathrm{I}_{-1}^{-}\right\rangle\right.$

$\left.<\mathrm{F}^{+}{ }_{1}, \mathrm{~F}^{+} 2, \ldots, \mathrm{F}^{+} ; \mathrm{F}^{0} ; \mathrm{F}_{-\mathrm{n}}^{-}, \ldots, \mathrm{F}_{-2}^{-}, \mathrm{F}_{-1}^{-}>\right)$

where we have multiple positive/neutral/negative degrees of T, I, and F respectively. 
12) The Neutrosophic Numbers have been introduced by W.B. Vasantha Kandasamy and F. Smarandache in 2003, which are numbers of the form $\mathrm{N}=a+b I$, where $a, b$ are real or complex numbers, while "I" is the indeterminacy part of the neutrosophic number N, such that $\mathrm{I}^{2}=\mathrm{I}$ and $\alpha \mathrm{I}+\beta \mathrm{I}=(\alpha+\beta) \mathrm{I}$.

Of course, indeterminacy "I" is different from the imaginary unit $i=\sqrt{-1}$.

In general one has $\mathrm{I}^{\mathrm{n}}=\mathrm{I}$ if $\mathrm{n}>0$, and it is undefined if $\mathrm{n} \leq 0$.

Also, Neutrosophic Refined Numbers were introduced (Smarandache, 2015) as: $a+b_{1} I_{1}+b_{2} I_{2}+\ldots+b_{m} I_{m}$, where $a, b_{1}, b_{2}, \ldots, b_{m}$ are real or complex numbers, while the $I_{1}$, $I_{2}, \ldots, I_{m}$ are types of indeterminacies, for $m \geq 1$.

The algebraic structures using neutrosophic numbers gave birth to the $\boldsymbol{I}$-Neutrosophic Algebraic Structures [see for example "neutrosophic groups", "neutrosophic rings", "neutrosophic vector space", "neutrosophic matrices, bimatrices, ..., n-matrices", etc.], introduced by W.B. Vasantha Kandasamy, F. Smarandache et al. since 2003.

Example of Neutrosophic Matrix: $\left[\begin{array}{ccc}1 & 2+\mathrm{I} & -5 \\ 0 & 1 / 3 & \mathrm{I} \\ -1+4 \mathrm{I} & 6 & 5 \mathrm{I}\end{array}\right]$.

Example of Neutrosophic Ring: $(\{a+b I$, with $a, b \in R\},+, \cdot)$, where of course $(a+b I)+(c+d I)=$ $(a+c)+(b+d) I$, and $(a+b I) \cdot(c+d I)=(a c)+(a d+b c+b d) I$.

Also, to Refined I-Neutrosophic Algebraic Structures, which are structures using sets of refined neutrosophic numbers.

13) Types of neutrosophic graphs (and trees):

13.a-c) Indeterminacy "I" led to the definition of the Neutrosophic Graphs (graphs which have: either at least one indeterminate edge, or at least one indeterminate vertex, or both some indeterminate edge and some indeterminate vertex), and Neutrosophic Trees (trees which have: either at least one indeterminate edge, or at least one indeterminate vertex, or both some indeterminate edge and some indeterminate vertex), which have many applications in social sciences.

Another type of neutrosophic graph is when at least one edge has a neutrosophic $(t, i, f)$ truthvalue.

As a consequence, the Neutrosophic Cognitive Maps (Vasantha \& Smarandache, 2003) and Neutrosophic Relational Maps (Vasantha \& Smarandache, 2004) are generalizations of fuzzy cognitive maps and respectively fuzzy relational maps, Neutrosophic Relational Equations (Vasantha \& Smarandache, 2004), Neutrosophic Relational Data (Wang, Smarandache, Sunderraman, Rogatko - 2008), etc.

A Neutrosophic Cognitive Map is a neutrosophic directed graph with concepts like policies, events etc. as vertices, and causalities or indeterminates as edges. It represents the causal relationship between concepts.

An edge is said indeterminate if we don't know if it is any relationship between the vertices it connects, or for a directed graph we don't know if it is a directly or inversely proportional relationship. We may write for such edge that $(t, i, f)=(0,1,0)$.

A vertex is indeterminate if we don't know what kind of vertex it is since we have incomplete information. We may write for such vertex that $(t, i, f)=(0,1,0)$.

Example of Neutrosophic Graph (edges $\mathrm{V}_{1} \mathrm{~V}_{3}, \mathrm{~V}_{1} \mathrm{~V}_{5}, \mathrm{~V}_{2} \mathrm{~V}_{3}$ are indeterminate and they are drawn as dotted): 


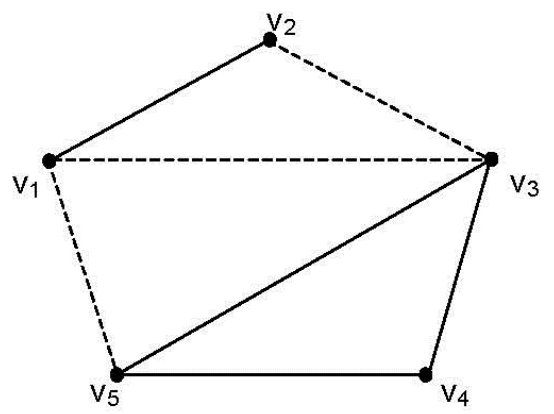

and its neutrosophic adjacency matrix is:

$$
\left[\begin{array}{lllll}
0 & 1 & \mathrm{I} & 0 & \mathrm{I} \\
1 & 0 & \mathrm{I} & 0 & 0 \\
\mathrm{I} & \mathrm{I} & 0 & 1 & 1 \\
0 & 0 & 1 & 0 & 1 \\
\mathrm{I} & 0 & 1 & 1 & 0
\end{array}\right]
$$

The edges mean: $0=$ no connection between vertices, $1=$ connection between vertices, $\mathrm{I}=$ indeterminate connection (not known if it is, or if it is not).

Such notions are not used in the fuzzy theory.

Example of Neutrosophic Cognitive Map (NCM), which is a generalization of the Fuzzy Cognitive Maps.

Let's have the following vertices:

$\mathrm{C}_{1}$ - Child Labor

$\mathrm{C}_{2}$ - Political Leaders

$\mathrm{C}_{3}$ - Good Teachers

$\mathrm{C}_{4}$ - Poverty

$\mathrm{C}_{5}$ - Industrialists

$\mathrm{C}_{6}$ - Public practicing/encouraging Child Labor

$\mathrm{C}_{7}$ - Good Non-Governmental Organizations (NGOs) 


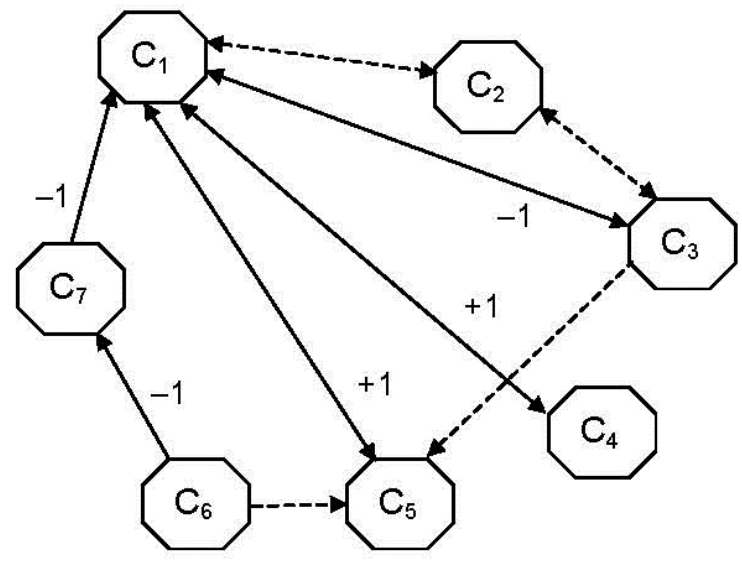

The corresponding neutrosophic adjacency matrix related to this neutrosophic cognitive map is:

$$
\left[\begin{array}{ccccccc}
0 & I & -1 & 1 & 1 & 0 & 0 \\
I & 0 & I & 0 & 0 & 0 & 0 \\
-1 & I & 0 & 0 & I & 0 & 0 \\
1 & 0 & 0 & 0 & 0 & 0 & 0 \\
1 & 0 & 0 & 0 & 0 & 0 & 0 \\
0 & 0 & 0 & 0 & I & 0 & -1 \\
-1 & 0 & 0 & 0 & 0 & 0 & 0
\end{array}\right]
$$

The edges mean: $0=$ no connection between vertices, $1=$ directly proportional connection, -1 = inversely proportionally connection, and $\mathrm{I}=$ indeterminate connection (not knowing what kind of relationship is between the vertices that the edge connects).

13.d) Another type of neutrosophic graphs (and trees) [Smarandache, 2015]:

An edge of a graph, let's say from A to B (i.e. how A influences B),

may have a neutrosophic value $(t, i, f)$,

where $t$ means the positive influence of $A$ on $B$,

i means the indeterminate influence of $\mathrm{A}$ on $\mathrm{B}$,

and $\mathrm{f}$ means the negative influence of $\mathrm{A}$ on $\mathrm{B}$.

Then, if we have, let's say: A->B->C such that A->B has the neutrosophic value $\left(t_{1}, i_{1}, f_{1}\right)$ and $B->C$ has the neutrosophic value $\left(t_{2}, i_{2}, f_{2}\right)$, then $A->C$ has the neutrosophic value $\left(t_{1}, i_{1}, f_{1}\right) \wedge\left(t_{2}, i_{2} . f_{2}\right)$, where $\Lambda$ is the AND neutrosophic operator.

13.e) Also, again a different type of graph: we can consider a vertex A as: t\% belonging/membership to the graph, $\mathrm{i} \%$ indeterminate membership to the graph, and $\mathrm{f} \%$ nonmembership

to the graph.

13.f) Any of the previous types of graphs (or trees) put together. 
13.g) Tripolar (and Multipolar) Graph, which is a graph whose vertexes or edges have the form $\left(\left\langle\mathrm{T}^{+}, \mathrm{T}^{0}, \mathrm{~T}^{-}\right\rangle,\left\langle\mathrm{I}^{+}, \mathrm{I}^{0}, \mathrm{I}^{-}\right\rangle,\left\langle\mathrm{F}^{+}, \mathrm{F}^{0}, \mathrm{~F}^{-}\right\rangle\right)$and respectively: $\left(\left\langle\mathrm{T}^{+}{ }_{\mathrm{j}}, \mathrm{T}^{0}, \mathrm{~T}^{-} \mathrm{j}^{-},\left\langle\mathrm{I}^{+} \mathrm{j}, \mathrm{I}^{0}, \mathrm{I}_{\mathrm{j}}^{-}\right\rangle,\left\langle\mathrm{F}^{+}{ }_{\mathrm{j}}\right.\right.\right.$, $\left.\mathrm{F}^{0}, \mathrm{~F}_{\mathrm{j}}^{-}>\right)$.

14) The Neutrosophic Probability (NP), introduced in 1995, was extended and developed as a generalization of the classical and imprecise probabilities [Smarandache (2013)]. NP of an event $\mathscr{E}$ is the chance that event $\mathcal{E}$ occurs, the chance that event $\mathscr{E}$ doesn't occur, and the chance of indeterminacy (not knowing if the event $\mathscr{E}$ occurs or not).

In classical probability $\mathrm{n}_{\text {sup }} \leq 1$, while in neutrosophic probability $\mathrm{n}_{\text {sup }} \leq 3^{+}$.

In imprecise probability: the probability of an event is a subset $\mathrm{T}$ in $[0,1]$, not a number $\mathrm{p}$ in $[0,1]$, what's left is supposed to be the opposite, subset $\mathrm{F}$ (also from the unit interval $[0,1]$ ); there is no indeterminate subset I in imprecise probability.

In neutrosophic probability one has, besides randomness, indeterminacy due to construction materials and shapes of the probability elements and space.

In consequence, neutrosophic probability deals with two types of variables: random variables and indeterminacy variables, and two types of processes: stochastic process and respectively indeterminate process.

15) And consequently the Neutrosophic Statistics, introduced in 1995 and developed in [Smarandache (2014)], which is the analysis of the neutrosophic events.

Neutrosophic Statistics means statistical analysis of population or sample that has indeterminate (imprecise, ambiguous, vague, incomplete, unknown) data. For example, the population or sample size might not be exactly determinate because of some individuals that partially belong to the population or sample, and partially they do not belong, or individuals whose appurtenance is completely unknown. Also, there are population or sample individuals whose data could be indeterminate. It is possible to define the neutrosophic statistics in many ways, because there are various types of indeterminacies, depending on the problem to solve.

Neutrosophic statistics deals with neutrosophic numbers, neutrosophic probability distribution, neutrosophic estimation, neutrosophic regression.

The function that models the neutrosophic probability of a random variable $\mathrm{x}$ is called neutrosophic distribution: $\mathrm{NP}(\mathrm{x})=(\mathrm{T}(\mathrm{x}), \mathrm{I}(\mathrm{x}), \mathrm{F}(\mathrm{x}))$, where $\mathrm{T}(\mathrm{x})$ represents the probability that value $\mathrm{x}$ occurs, $\mathrm{F}(\mathrm{x})$ represents the probability that value $\mathrm{x}$ does not occur, and $\mathrm{I}(\mathrm{x})$ represents the indeterminate / unknown probability of value $\mathrm{x}$.

16) Also, Neutrosophic Measure and Neutrosophic Integral were introduced [Smarandache (2013)].

17) Neutrosophy [Smarandache (1995)] opened a new field in philosophy.

Neutrosophy is a new branch of philosophy that studies the origin, nature, and scope of neutralities, as well as their interactions with different ideational spectra.

This theory considers every notion or idea $\langle A>$ together with its opposite or negation $<$ Anti$\mathrm{A}>$ and the spectrum of "neutralities" $<$ Neut-A $>$ (i.e. notions or ideas located between the two 
extremes, supporting neither $\langle$ A $\rangle$ nor $\langle$ Anti-A $\rangle$ ). The $\langle$ Neut-A $\rangle$ and $\langle$ Anti-A $\rangle$ ideas together are referred to as $\langle$ Non-A $\rangle$.

According to this theory every idea $\langle\mathrm{A}\rangle$ tends to be neutralized and balanced by $\langle$ Anti-A $\rangle$ and $<$ Non-A $>$ ideas - as a state of equilibrium.

In a classical way $\langle\mathrm{A}\rangle,\langle$ Neut-A $\rangle,\langle$ Anti-A $>$ are disjoint two by two.

But, since in many cases the borders between notions are vague, imprecise, Sorites, it is possible that $\langle\mathrm{A}\rangle$, $\langle$ Neut-A $\rangle$, $\langle$ Anti-A $\rangle$ (and $\langle$ Non-A $\rangle$ of course) have common parts two by two as well.

Neutrosophy is the base of neutrosophic logic, neutrosophic set, neutrosophic probability and statistics used in engineering applications (especially for software and information fusion), medicine, military, cybernetics, physics.

We have extended dialectics (based on the opposites $\langle\mathrm{A}\rangle$ and $\langle$ antiA $\rangle$ ) to neutrosophy (based on $\langle\mathrm{A}\rangle$, $\langle$ antiA $\rangle$ and $\langle$ neutA $\rangle$.

18) In consequence, we extended the thesis-antithesis-synthesis to thesis-antithesisneutrothesis-neutrosynthesis [Smarandache, 2015].

19) Neutrosophy extended the Lupasco-Nicolescu's Law of Included Middle to the Law of Included Multiple-Middle [Smarandache, 2014] in accordance with the n-valued refined neutrosophic logic.

20) Smarandache (2015) introduced the Neutrosophic Axiomatic System and Neutrosophic Deducibility.

21) Then he introduced the (t, i, f)-Neutrosophic Structure (2015), which is a structure whose space, or at least one of its axioms (laws), has some indeterminacy of the form (t, $\mathrm{i}, \mathrm{f})$ $\neq(1,0,0)$.

Also, we defined the combined $(t, i, f)$-I-Neutrosophic Algebraic Structures, i.e. algebraic structures based on neutrosophic numbers of the form $a+b I$, but also having some indeterminacy [ of the form $(t, i, f) \neq(1,0,0)]$ related to the structure space (i.e. elements which only partially belong to the space, or elements we know nothing if they belong to the space or not) or indeterminacy $\quad[$ of the form $(t, i, f) \neq(1,0,0)]$ related to at least one axiom (or law) acting on the structure space) .

Even more, we generalized them to Refined $(t, i, f)$ - Refined I-Neutrosophic Algebraic Structures, or $\left(t_{j}, i_{k}, f_{l}\right)$ - $i_{s}$-Neutrosophic Algebraic Structures; where $t_{j}$ means that $t$ has been refined to $j$ subcomponents $t_{1}, t_{2}, \ldots, t_{j}$; similarly for $i_{k}, f_{l}$ and respectively $i_{s}$. 
22) Smarandache - Ali introduced the Neutrosophic Triplet Structures.

A Neutrosophic Triplet, is a triplet of the form:

$$
<a, \operatorname{neut}(a) \text {, and } \operatorname{anti}(a)>
$$

where neut(a) is the neutral of a, i.e. an element (different from the identity element of the operation $*$ ) such that $a^{*}$ neut $(\mathrm{a})=\operatorname{neut}(\mathrm{a}) * \mathrm{a}=\mathrm{a}$,

while anti(a) is the opposite of a, i.e. an element such that $a^{*} \operatorname{anti}(a)=\operatorname{anti}(a) * a=\operatorname{neut}(a)$. Neutrosophy means not only indeterminacy, but also neutral (i.e. neither true nor false).

For example we can have neutrosophic triplet semigroups, neutrosophic triplet loops, etc.

We went further and extended our neutrosophic triplet $<$ a, neut(a), anti(a) $>$ to a

\section{$\boldsymbol{m}$-valued refined neutrosophic triplet,}

in a similar way as it was done for $\mathrm{T}_{1}, \mathrm{~T}_{2}, \ldots ; \mathrm{I}_{1}, \mathrm{I}_{2}, \ldots ; \mathrm{F}_{1}, \mathrm{~F}_{2}, \ldots$ (i.e. the refinement of neutrosophic components).

It will work in some cases, depending on the composition law *. It depends on each * how many neutrals and anti's there is for each element "a".

We may have an m-tuple with respect to the element " $a$ " in the following way:

( a; $\left.\operatorname{neut}_{1}(\mathrm{a}), \operatorname{neut}_{2}(\mathrm{a}), \ldots, \operatorname{neut}_{\mathrm{p}}(\mathrm{a}) ; \operatorname{anti}_{1}(\mathrm{a}), \operatorname{anti}_{2}(\mathrm{a}), \ldots, \operatorname{anti}_{\mathrm{p}}(\mathrm{a})\right)$,

where $\mathrm{m}=1+2 \mathrm{p}$,

such that:

- all neut $1(\mathrm{a})$, neut $_{2}(\mathrm{a}), \ldots$, neut $_{\mathrm{p}}(\mathrm{a})$ are distinct two by two, and each one is different from the unitary element with respect to the composition law *;

- also

$a^{*} \operatorname{neut}_{1}(\mathrm{a})=\operatorname{neut}_{1}(\mathrm{a}) * \mathrm{a}=\mathrm{a}$

$a^{*} \operatorname{neut}_{2}(a)=\operatorname{neut}_{2}(a) * a=a$

$a^{*} \operatorname{neut}_{\mathrm{p}}(\mathrm{a})=\operatorname{neut}_{\mathrm{p}}(\mathrm{a}) * \mathrm{*}=\mathrm{a}$

- and

$a^{*} \operatorname{anti}_{1}(a)=\operatorname{anti}_{1}(a) * a=\operatorname{neut}_{1}(a)$

$a^{*} \operatorname{anti}_{2}(a)=\operatorname{anti}_{2}(a) * a=\operatorname{neut}_{2}(a)$

$a^{*} \operatorname{anti}_{\mathrm{p}}(\mathrm{a})=\operatorname{anti}_{\mathrm{p}}(\mathrm{a}) * \mathrm{a}=$ neut $_{\mathrm{p}}(\mathrm{a})$

- where all anti $(a)$, anti $2(a), \ldots$, antip $(a)$ are distinct two by two, and in case when there are duplicates, the duplicates are discarded.

\section{References on Neutrosophics}

1. L. A. Zadeh, Fuzzy Sets. Information and Control, 1965, 8(5): 338- 353.

2. K. Atanassov, Intuitionistic fuzzy sets. Fuzzy Sets and Systems, 1986, 20: 87-96.

3. F. Smarandache, Neutrosophy. Neutrosophic Probability, Set, and Logic. Amer. Res. Press, Rehoboth, USA, 105 p., 1998, 2000, 2002, 2005, 2006.

4. K. T. Atanassov (1999), Intuitionistic Fuzzy Sets, Physica-Verlag, Heidelberg, N.Y.

5. N. Belnap (1977), A Useful Four-Valued Logic, Modern Uses of Multiple-Valued Logics (D. Reidel, ed.), 8-37.

6. H. Bustince, P. Burillo (1996), Vague Sets are Intiotionistic Fuzzy Sets, Fuzzy Sets and Systems, 79, 403-405.

7. C. Cornelis, K. T. Atanassov, E. E. Kerre (2003), Intuitionistic Fuzzy Sets and 
Interval-Valued Fuzzy Sets: a Critical Comparaison, Proceedings of the Third Conference of the European Society for Fuzzy Logic and Technology, EUSFLAT 2003, September 10-12, 2003, Zittau, Germany; University of Applied Sciences at Zittau/Goerlitz, 159-163.

8. J. L. Deng (1989), Introduction to Grey System Theory, J. Grey Systems, 1, 1-24. [] G. Deschrijver, E. E. Kerre (2003), On the Relationship between some Extensions of Fuzzy Set Theory, Fuzzy Sets and Systems, 133, 227-235.

9. Jean Dezert, Open Questions to Neutrosophic Inferences, Multiple-Valued Logic / An International Journal, Vol. 8, No. 3, 439-472, June 2002.

10. D. Dubois, S. Gottwald, P. Hajek, H. Prade, Terminological difficulties in fuzzy set theory - The case of intuitionistic fuzzy sets, Fuzzy Sets and Systems, 156 (2005), $585-491$.

11. W. L. Gau, D. J. Buehrer (1993), Vague Sets, IEEE Trans. Systems Man Cybernet, 23 (2), 610-614.

12. M. Gehrke, C. Walker, E. Walker (1996), Some Comments on Interval-Valued Fuzzy Sets, Int. Journal of Intelligent Systems, 11 (10), 751-759.

13. J. Goguen (1967), L-fuzzy Sets, J. Math. Anal. Appl., 18, 145-174.

14. R. Sambuc (1975), Fonctions $\Phi$-floues. Application l'Aide au Diagnostic en Pathologie Thyroidienne, Ph. D. Thesis, Univ. Marseille, France.

15. F. Smarandache (2003), Definition of Neutrosophic Logic - A Generalization of the Intuitionistic Fuzzy Logic, Proceedings of the Third Conference of the European Society for Fuzzy Logic and Technology, EUSFLAT 2003, September 10-12, 2003, Zittau, Germany; University of Applied Sciences at Zittau/Goerlitz, 141-146.

16. F. Smarandache (2002a), A Unifying Field in Logics: Neutrosophic Logic, in Multiple-

Valued Logic / An International Journal, Vol. 8, No. 3, 385-438, 2002, www.gallup.unm.edu/ smarandache/eBook-neutrosophics4.pdf.

17. F. Smarandache (2002b), Neutrosophy, A New Branch of Philosophy, in MultipleValued Logic / An International Journal, Vol. 8, No. 3, 297-384, 2002. This whole issue of this journal is dedicated to Neutrosophy and Neutrosophic Logic.

18. F. Smarandache (2002c), editor, Proceedings of the First International Conference on Neutrosophy, Neutrosophic Logic, Neutrosophic Set, Neutrosophic Probability and Statistics, University of New Mexico, Gallup Campus, Xiquan, Phoenix, 147 p., 2002, www.gallup.unm.edu/ smarandache/NeutrosophicProceedings.pdf.

19. F. Smarandache (2013), Introduction to Neutrosophic Measure, Neutrosophic Integral and Neutrosophic Probability, Sitech, 2013; http://fs.gallup.unm.edu/NeutrosophicMeasureIntegralProbability.pdf.

20. F. Smarandache (2014), Introduction to Neutrosophic Statistics, Sitech, 2014, http://fs.gallup.unm.edu/NeutrosophicStatistics.pdf.

21. Eulalia Szmidt, Janusz Kacprzyk, Distances between fuzzy sets, Fuzzy Sets and Systems, 114 (2000), 505-518.

22. P. Walley (1991), Statistical Reasoning with Imprecise Probability, Chapman/Hall.

23. L. Zadeh (2003), From Search Engines to Question-Answering Systems - The Need For New Tools, Proceedings of the Third Conference of the European Society for Fuzzy Logic and Technology, 3-5, Zittau, Germany. 
Previous versions of this article were published as follows:

- Florentin Smarandache, Neutrosophic Set, A Generalization of the Intuitionistic Fuzzy Set, International Journal of Pure and Applied Mathematics, Vol. 24, No. 3, 287-297, 2005;

a. also in Proceedings of 2006 IEEE International Conference on Granular

Computing, edited by Yan-Qing Zhang and Tsau Young Lin, Georgia State University, Atlanta, pp. 38-42, 2006;

b. second version in Journal of Defense Resources Management, Brasov, Romania, No. 1, 107-116, 2010.

- Florentin Smarandache, A Geometric Interpretation of the Neutrosophic Set - A Generalization of the Intuitionistic Fuzzy Set, 2011 IEEE International Conference on Granular Computing, edited by Tzung-Pei Hong, Yasuo Kudo, Mineichi Kudo, TsauYoung Lin, Been-Chian Chien, Shyue-Liang Wang, Masahiro Inuiguchi, GuiLong Liu, IEEE Computer Society, National University of Kaohsiung, Taiwan, 602-606, 8-10 November 2011. 